\title{
Herida por arma de fuego en cráneo
}

\author{
Injure caused by firearm in skull
}

José D. Charry', José F. Arango²

\section{Resumen}

Las heridas ocasionadas por proyectiles de armas de fuego son muy comunes en la práctica diaria de quienes atienden el neurotrauma, se presenta un caso exitoso de un paciente masculino de 22 años quien sufrió herida por proyectil de arma de fuego en cráneo, orificio de entrada en región parieto-occipital derecha, con exposición de masa encefálica y sin orifico de salida; al ingreso presentó Glasgow 12/15, somnolencia, disartria, desviación de la comisura labial a la izquierda, hemiparesia derecha; fue llevado a salas de cirugía donde le realizaron esquirlectomía y descompresión craneana temprana bilateral (temporo-parieto-occipital) como procedimiento control de daños, los resultados posoperatorios fueron sin complicaciones, evolución neurológica satisfactoria, y al egreso hospitalario el paciente presentó GOS de $4 / 5$.

Palabras clave: Lesión cerebral traumática, heridas por arma de fuego, lesiones penetrantes de cráneo.

\begin{abstract}
Wounds caused by projectiles of firearms are common in the daily practice of those who attend the neurotrauma. A successful case of a male patient aged 22 who suffered injury by gunfire in skull, inletoccurs inright parietal-occipital region, with exposed brain matter with out outlet; Glasgow $12 / 15$ was present at admission, drowsiness, dysarthria, left deviation of the corner of the mouth, righ the miparesis; the patient was taken to the operating room where he under wentes quillectomy and bilateral early cranial decompression (temporo-parietal-occipital) asdamage control procedure. Post operative results had no complications, satisfactory neurological outcome, and when discharging from hospital, the patient had GOS4/5.
\end{abstract}

Keywords: Traumatic brain injury, firearm wounds, penetrating head injuries.

\section{Introducción}

El Trauma craneoencefálico (TCE) severo es una patología de importancia a nivel global. Se han reportado incidencias cercanas a 200 casos por cada 100.000 habitantes a nivel mundial ${ }^{[1]}$. De acuerdo al estudio de la Organización Mundial de la Salud, sobre carga global de enfermedad publicado en el año $2010^{[2,3]}$, el trauma continúa siendo un problema de salud pública y genera una carga importante para los sistemas de salud en países latinoamericanos. En Colombia la carga global de las lesiones, impacta más en la población económicamente activa de sexo masculino entre 12 y 45 años; en el 2013 por ejemplo, se presentaron alrededor de 26.000 muertes por trauma y la gran mayoría de ellas estaban asociadas a violencia interpersonal; de estas lesiones, un gran porcentaje estuvieron asociadas a TCE severo tanto cerrado como penetrante ${ }^{[4]}$.

Las heridas ocasionadas por proyectil de arma de fuego, son comunes en la práctica diaria de quienes atienden el neurotrauma, siendo muchas de ellas de alta mortalidad ${ }^{[5,6]}$. Estas lesiones son más frecuentes en escenarios militares. Recientemente un enfoque agresivo de control de daños (CD) para el manejo de estas lesiones mediante la descompresión craneana temprana (DCT) ha sido utilizado por los neurocirujanos militares en áreas de combate en Irak y Afganistán. ${ }^{[7]}$ Este es el reporte de caso de un paciente masculino de 22 años quien sufrió herida por proyectil de arma de fuego en cráneo, con el objetivo de describir el manejo de DCT como procedimiento de CD en neurotrauma en el Hospital Universitario de Neiva

\section{Descripción del caso}

Paciente masculino de 22 años que ingresa al servicio de urgencias del Hospital Universitario de Neiva, Hernando Moncaleano Perdomo (HUHMP) por presentar herida por proyectil de arma de fuego (PAF) con orifico de entrada en la región temporo parietal derecha y sin orificio de salida; al ingreso el paciente estaba somnoliento con signos vitales de TA: 110/70, FC: 52, FR: 28, presentando desviación de la comisura labial izquierda, ptosis palpebral izquierda, hemiparesia derecha, pupilas de $2 \mathrm{~mm}$ de diámetro lentas a la luz, y escala de coma de Glasgow (ECG) $12 / 15$, con tendencia al deterioro neurológico por lo cual se deci-

Estudiante de Medicina, Facultad de Salud, Universidad Surcolombiana.

2 Neurocirujano, Hospital Universitario de Neiva, Hernando Moncaleano Perdomo.

Correspondencia: José Daniel Charry Cuéllar. Correo electrónico:danielcharry06@gmail.com

Recibido: 19/04/14-Revisado: 22/06/2014-Aceptado: 25/09/2014 


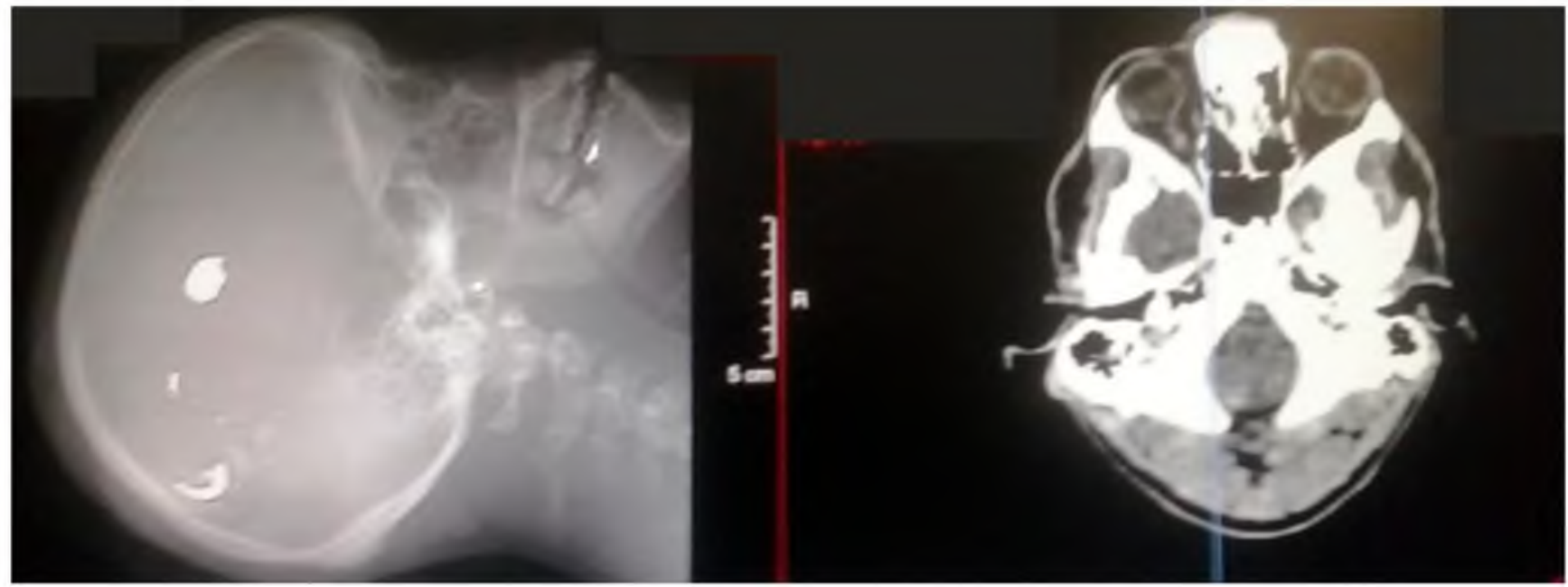

Figura 1. Proyección lateral de la Tomografía Computarizada donde se evidencia el ingreso y trayecto del proyectil a nivel temporo-parietal derecho.

dió realizar intubación orotraqueal y manejo anti edema cerebral con el uso de soluciones hipertónicas y medidas farmacológicas para evitar maniobras de valsalva; el paciente fue trasladado a imágenes diagnosticas donde le realizaron tomografía computarizada (TC) (Figura 1 y 2) que evidenció el trayecto del proyectil. Neurocirugía valoro el paciente, describe las lesiones que se observan en la tomografía (Figura 3), decide llevarlo a salas de cirugía donde realizan esquirlectomía más craniectomía

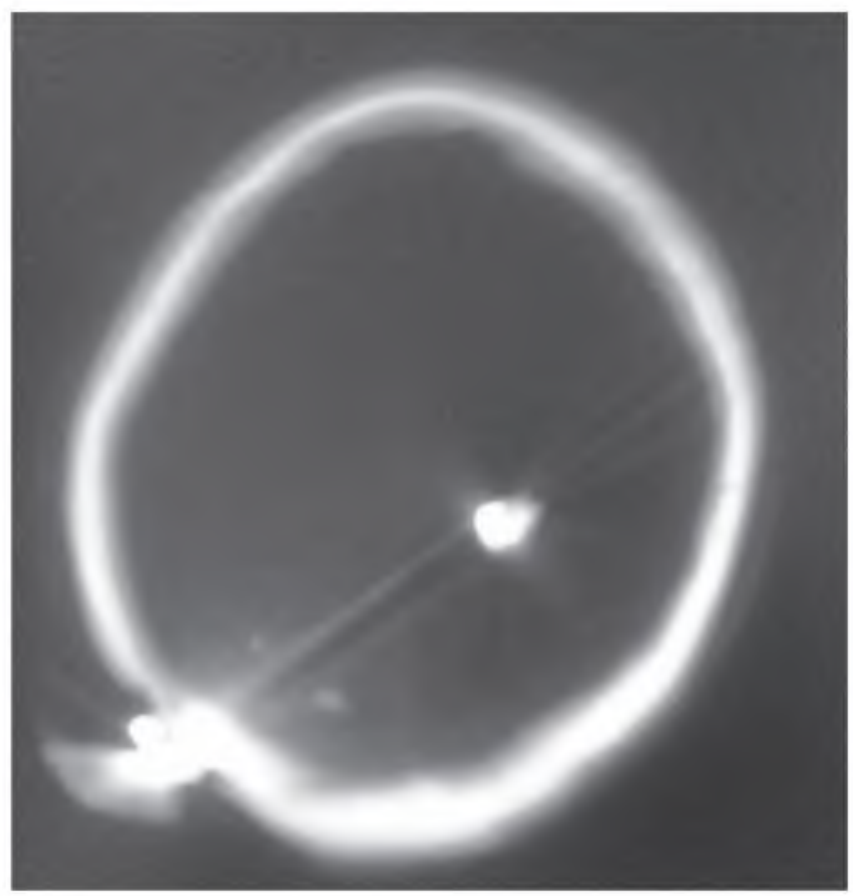

Figura 2. Tomografía computarizada en corte axial y proyección de la ventana ósea, donde se observa el ingreso del proyectil por el lado temporo - parietal derecho y se aloja a nivel parietal izquierdo. Se observan las esquirlas en el orificio de ingreso del proyectil descompresiva temprana bilateral (temporo-parieto-occipital) y ventriculostomía. Durante el procedimiento quirúrgico se logró extraer el proyectil, el cual entró bajo custodia inmediata de medicina legal.

E1 posoperatorio inmediato se llevó a cabo en la Unidad de Cuidados Intensivos (UCI) con monitoreo de presion intracraneal (PIC) la cual no registro curvas de hipertensión endocraneana, adicional a esto el manejo en la UCI se orientó a sedación y

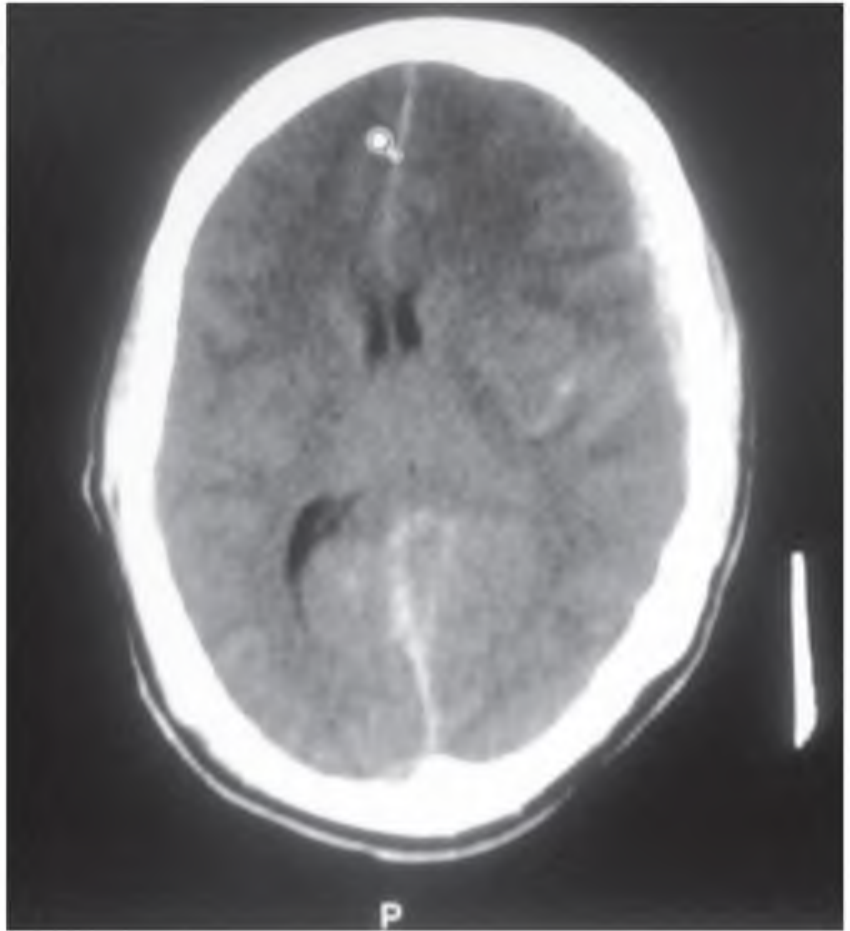

Figura 3. Tomografía computarizada de cráneo, corte axial, en proyección de parénquima cerebral donde se evidencia desviación de línea media, cisternas de las bases obliteradas, hemorragia subaracnoidea, edema cerebral. 


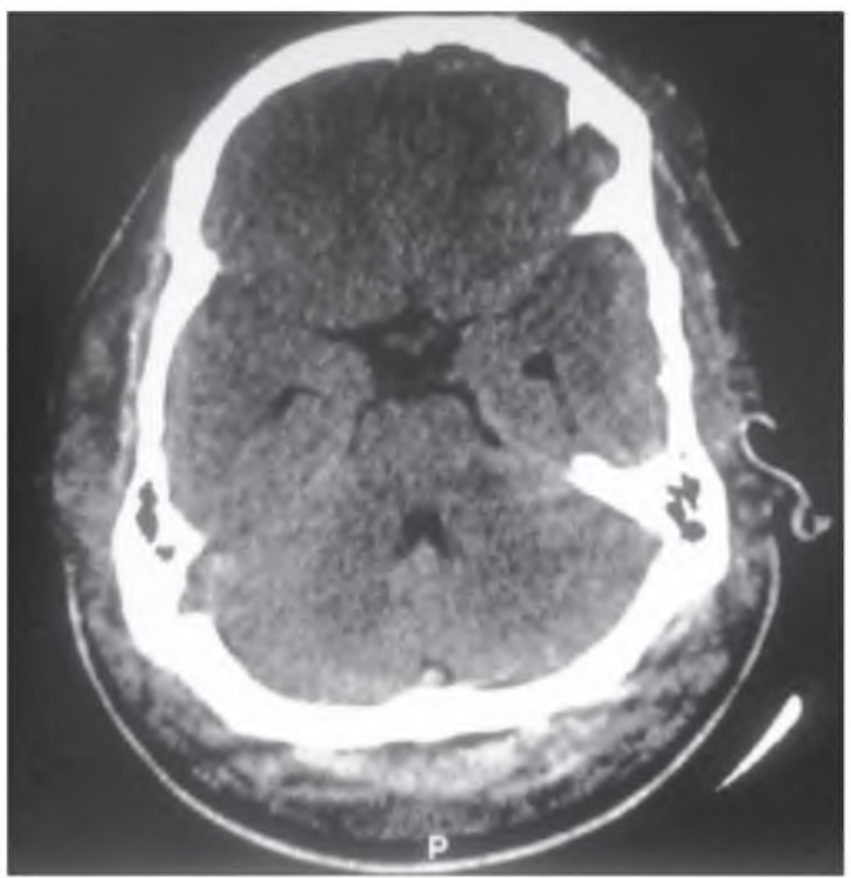

Figura 4. Tomografía computarizada de cráneo, corte axial, en proyección de parénquima cerebral de control realizada a las 48 horas del ingreso a la institución. Acá se evidencia que no hay desviación de línea media, cisternas de las bases están permeables y ha disminuido el edema cerebral.

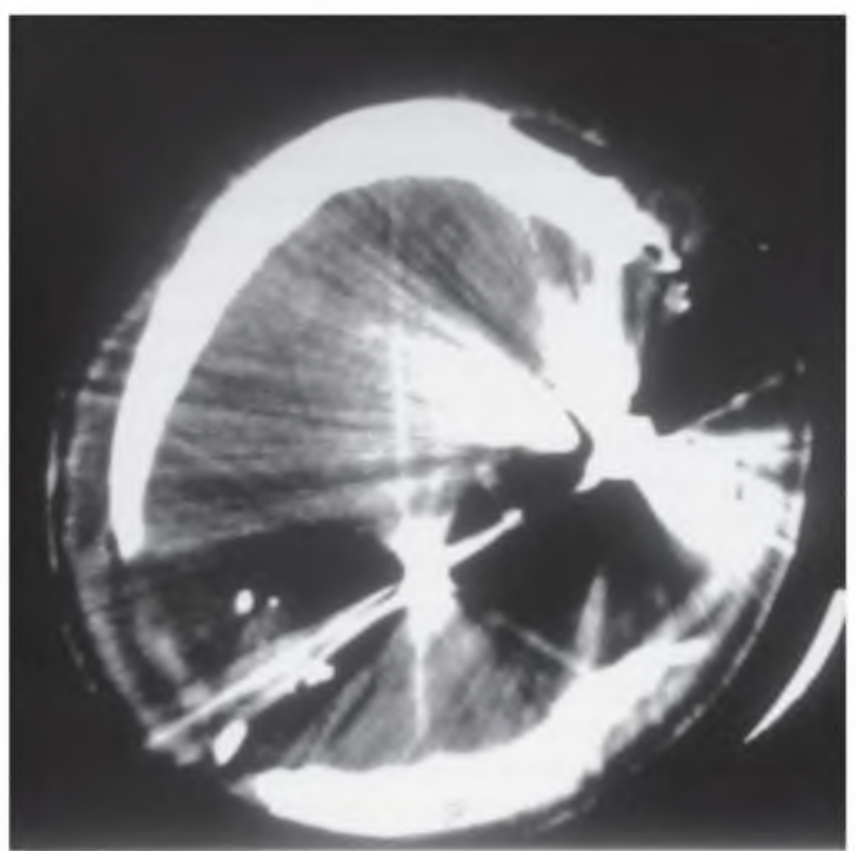

Figura 5. Tomografía computarizada de cráneo, corte axial, realizada a las 48 horas del ingreso a la institución. Acá se evidencia el área de craniectomía descompresiva (temporo - parieto - occipital) bilateral, algunos artificios. analgesia, junto a medidas anti edema cerebral, a las 48 horas se le realizó TC Cerebral de control (Figura 4) la cual evidenció disminución del edema cerebral, sin hemorragias, línea media conservada, cisternas de la base abiertas y laceración cortical en hemisferios, (Figura 5) se observa la craniectomía descompresiva bilateral.

E1 paciente presenta evolución neurológica satisfactoria durante su estancia en la UCI con ECG de 13/15, reflejos de tallo cerebral conservados (reflejo corneano presente, reflejo nauseoso presente), pupilas isocoricas normorreactivas a la luz, moviliza las 4 extremidades, se egresa de la unidad de cuidados intensivos al 6 día para recuperación en el piso de neurocirugía.

Después de 28 días de la lesión cerebral traumática por herida por arma de fuego el paciente egresó de la institución con ECG de 14/15, movilizando las 4 extremidades, pupilas isocoricas normo reactivas a la luz, reflejos musculotendinosos $++/++++$, fuerza muscular $5 / 5$ en las cuatro extremidades, el paciente no presenta déficit motor ni sensitivo, buena recuperación neurológica de acuerdo a la escala de pronóstico de glasgow (GOS) la cual establece una puntuación de 1 a 5 donde los pacientes que tengan alguna puntuación 1-3 se catalogan con resultado neurológico desfavorable, y los pacientes con puntuación 4-5 resultado neurológico favorable. En este caso el paciente egresa de la institución con GOS 4/5, adicional se le dan las recomendaciones médica y signos de alarma para consultar nuevamente a la institución.

\section{Discusión}

El manejo de las lesiones ocasionadas por proyectiles de arma de fuego en cráneo ha sido reorientado en los últimos años. Generalmente el tratamiento conservador de estos pacientes se realizaba porque este tipo de lesiones se caracterizaban por ser de mal pronóstico en estudios de la década de $\operatorname{los} 80^{[8]}$. En base a la experiencia obtenida recientemente por neurocirujanos militares en áreas de conflicto en el medio oriente (Irak y Afganistán principalmente), donde la posibilidad de neuromonitoría avanzada para tomar decisiones quirúrgicas era casi inexistente, se tuvo en cuenta la posibilidad de realizar un manejo quirúrgico más agresivo. Inicialmente este manejo era básicamente craneotomía, drenaje de hematomas, esquirlectomía y desbridamiento del tejido necrótico ${ }^{[9]}$

Años más tarde aparece el termino de control de daños que consiste en realizar una descompresión craneana temprana ${ }^{[10,11]}$. Esta estrategia ha sido promulgada como una importante opción para mejorar sobrevida y disminuir discapacidad ${ }^{[12,13]}$. Esta tendencia se observó en el caso que se presenta, puesto que la sobrevida y el pronóstico de vida para el paciente fueron favorable.

Las heridas por proyectil de arma de fuego, son lesiones extremadamente severas y su alta morbimortalidad está ligada a diferentes factores como lo son trayectoria transventricular, lesiones que comprometen más de un lóbulo, heridas que atraviesan línea media, proyectiles múltiples que cruzan línea media, borramiento de las cisternas de la base, y hemorragia subaracnoidea perimesencefálica. ${ }^{[14,15]}$ En este caso se observó el compromiso del lóbulo temporal y parietal y el trayecto del proyectil que cruza línea media.

Cabe resaltar que la mayoría de los estudios realizados en pacientes con lesiones ocasionadas por proyectiles de arma de fuego en cráneo, se han realizado en centros donde existe la posibilidad de monitoria neurológica constante en la unidad de cuidado intensivo $^{[15,16]}$. En nuestro caso, el centro donde se realizó el 
manejo de este paciente, no cuenta con la posibilidad de neuromonitoría avanzada. Las secuelas del caso reportado difieren de manera considerables a las reportadas en la literatura ${ }^{[17-19]}$. La evolución sin secuelas se atribuye al manejo de control de daños en neurotrauma mediante la descompresión craneana temprana, la atención inmediata de la herida, el tratamiento antimicrobiano y el manejo adecuado en la unidad de cuidado intensivo mediante el correcto esquema de medidas anti edema cerebral. En la actualidad el paciente no presenta déficit neurológico, y con favorable pronostico neurológico.

\section{Conclusión}

El control de daños para neurotrauma con una craniectomía descompresiva temprana es una opción para mejorar la supervivencia y el resultado neurológico favorable en pacientes con TCE penetrante. Este caso presento sobrevida y buen resultado neurológico favorable, cabe resaltar que se deben realizar estudios clínicos aleatorizados que permitan establecer el manejo ideal para este grupo de pacientes.

\section{Referencias}

1. Byass $P$, de Courten $M$, Graham WJ, Laflamme L, McCaw-Binns A, et al. (2013) Reflections on the Global Burden of Disease 2010 Estimates. PLoS Medicine 10(7):e1001477.

2. Horton R. GBD 2010: understanding disease, injury, and risk. Lancet. 2012 Dec 15;380(9859):2053-2054.

3. Norton R, Kobusingye $O$. Injuries. N Engl J Med. 2013May 2;368(18):1723-1730.

4. De la Hoz GA. Comportamiento del homicidio en Colombia, 2013. Forensis, 2013:79-125.

5. Coughlan MD, Fieggen AG, Semple PL, Peter JC. Craniocerebral gunshot injuries in children. Childs Nerv. Syst. (2003) 19:348-352.

6. Karabagli H. Spontaneous movement of bullets in the interhemispheric region. Ped. Neurosurg. (2005)41:148150.

7. Gurdjian SE. The treatment of penetrating wounds of the brain sustained in warfare. J. Neurosurgery, 2004;39:157-167.

8. Stone JL, Lichtor T, Fitzgerald LF. Gunshot wounds to the head in civilian practice. Neurosurgery. 1995 Dec;37(6):1104-1110.
9. Levy ML. Outcome prediction following penetrating craniocerebral injury in a civilian population: aggressive surgical management in patients with admission Glasgow Coma Scale scores of 6 to 15. Neurosurg Focus. 2000Jan 15;8(1).

10. Smith JE, Kehoe A, Harrisson SE, Russell R, Midwinter $M$. Outcome of penetrating intracranial injuries in a military setting. Injury. 2014;45(5):874-878.

11. Rubiano $A M$, Villarreal W, Hakim EJ, Aristizabal J, Hakim F, Diez JC, Peña G, Puyana JC. Early decompressive craniectomy for neurotrauma: an institutional experience.Ulus Travma Acil Cerrahi Derg. 2009 Jan; 15(1):28-38.

12. Bell RS, Mossop CM, Dirks MS, Stephens FL, Mulligan L, Ecker R, Neal CJ, Kumar A, Tigno T, Armonda RA. Early decompressive craniectomy for severe penetrating and closed head injury during wartime. Neurosurg Focus. 2010,28(5).

13. Gouello G, Hamel O, Asehnoune K, BordE, Robert R, Buffenoir K. Study of the Long-Term Results of Decompressive Craniectomy after Severe Traumatic Brain Injury Based on a Series of 60 Consecutive Cases. The Scientific World Journal Volume 2014, Article ID 207585, 10 pages.

14. Rubiano AM, Sarmiento FA, Pérez AF: Guías de Manejo Integral del Trauma Craneoencefálico en Áreas de Combate y Manejo de Heridas por Proyectil de Arma de Fuego en Cráneo; en: Rubiano AM, Pérez R: Neurotrauma y Neurointensivismo. 1@ Ed. Editorial Distribuna. Bogotá; 2007. pp 231-244.

15. Mackeric $Z$, Gal P Unusual penetrating head injury in children: personal experience and review of literature. Childs Nervous System (2009)25:909- 913.

16. Aarabi B. Management of Missile Head Wounds. Neurosurgery Quarterly (2003) 13(2):87-104.

17. Nagib, M.G., Rockswold, G.L., Sherman, R.S., Lagaard, M.W. 1986. Civilian gunshot wounds to the brain: Prognosis and management. Neurosurgery 18:533-537.

18. Jennett, B., Bond, M. 1975. Assessment of outcome after severe brain damage, a practical scale. Lancet 1:480-484.

19. Gressot LV, Chamoun RB, Patel AJ, Valadka AB, Suki D, Robertson CS, Gopinath SP Predictors of outcome in civilians with gunshot wounds to the head upon presentation. J Neurosurg. 2014Sep;121(3):645-652. 